\section{Kraftstoff, Wärme oder Strom aus Stroh und Waldrestholz - ein systemanalytischer Vergleich}

\author{
von Ludwig Leible, Stefan Kälber, Gunnar \\ Kappler, Stephan Lange, Eberhard Nieke, \\ Peter Proplesch, Detlev Wintzer und Beate \\ Fürniß, ITAS
}

Stroh und Waldrestholz bieten ein großes und kostengünstiges Potenzial für eine energetische Nutzung zur Wärme-, Stromund Kraftstoffgewinnung. Der Vergleich zeigt, dass gemessen an den Gestehungsund $\mathrm{CO}_{2}$-Minderungskosten die Kraftstoffgewinnung unter derzeitigen Rahmenbedingungen den höchsten Subventionsbedarf hat. Durch Nutzung technischer Fortschritte lässt sich dieser Nachteil sicherlich reduzieren, deshalb sollte - insbesondere unter Vorsorge-Gesichtspunkten - die Forschung und Demonstration in diesem Bereich intensiviert werden.

\section{Einleitung}

Aktuelle politische Ziele und Vorgaben auf EU- und nationaler Ebene, wie z. B. die Gemeinschaftsstrategie und der Aktionsplan der EU zur verstärkten Nutzung erneuerbarer Energieträger und die Biokraftstoffrichtlinie oder in Deutschland das Erneuerbare-Energien-Gesetz (EEG), zielen darauf ab, den Anteil erneuerbarer Energieträger an der Energieversorgung deutlich zu erhöhen. Dies betrifft sowohl die Bereitstellung von Wärme und Strom, aber auch von Kraftstoffen. Hierbei werden hohe Erwartungen v. a. an die energetische Nutzung von Biomasse und insbesondere an biogene Reststoffe geknüpft.

Um eine nachhaltige, sichere und bezahlbare Energieversorgung zu gewährleisten, hat sich die europäische Energiepolitik bis 2010 insbesondere folgende Ziele gesetzt:

- Erhöhung des Anteils der erneuerbaren Energien auf $12 \%$ des Primärenergieverbrauchs,

- Erhöhung des Anteils der Elektrizität aus erneuerbaren Energiequellen auf $22 \%$ der Stromproduktion,
- Erhöhung des Anteils der Biokraftstoffe auf $5,75 \%$ im Kraftstoffmarkt,

- Reduzierung der Treibhausgasemissionen um 8 \% (Bezugsjahr: 1990).

Wird bei den Biokraftstoffen die Zielsetzung der EU-Kommission ernst genommen, bis 2010 ihren Beitrag an der Kraftstoffversorgung auf 5,75\% und längerfristig auf $20 \% \mathrm{zu}$ erhöhen - 2005 lag der Anteil in Deutschland bei 3,4\%-, dann müssen hierzu auch Lignozelluloseträger (wie z. B. Stroh oder Waldrestholz) herangezogen werden.

Ausgehend vom „Biomass-to-Liquid“Konzept des Forschungszentrums Karlsruhe werden in diesem Beitrag Ergebnisse zur Gewinnung von Fischer-Tropsch-Kraftstoffen (FT-Kraftstoff) aus Stroh und Waldrestholz in den Mittelpunkt gestellt. Zunächst wird jedoch - in Relation zum insgesamt verfügbaren Aufkommen an biogenen Rest- und Abfallstoffen - dargestellt, welche energetisch nutzbaren Potenziale an Stroh und Waldrestholz in Deutschland und Baden-Württemberg zur Verfügung stehen. Der gezielte Anbau von Biomasse als Energieträger wird dabei nicht betrachtet. Anschließend wird am Beispiel von Baden-Württemberg in starker regionaler Differenzierung und mit Blick auf die Biomasseversorgung von Großanlagen zur Kraftstoffproduktion illustriert, welche Bedeutung der Logistik zur Biomassebereitstellung zuzumessen ist. Nach einem Überblick zu den energetischen Nutzungspfaden von Biomasse und biogenen Reststoffen und Abfällen werden Ergebnisse zur Produktion von FTKraftstoffen aus Stroh und Waldrestholz vorgestellt - neben technischen Erläuterungen zum Konzept insbesondere Ergebnisse zu den Produktionskosten. Daran schließt der Vergleich zu den Gestehungskosten der Wärmeund Stromgewinnung aus Stroh und Waldrestholz an. Im letzten Teil des Beitrags werden die Ergebnisse zur Bereitstellung von FischerTropsch-Kraftstoff aus Stroh und Waldrestholz anhand der Kennwerte „Gestehungskosten" und " $\mathrm{CO}_{2}$-Minderungskosten" in Vergleich zur Wärme- und Stromgewinnung gesetzt und bewertet. 


\section{Biomasseaufkommen und Logistik}

Das jährlich verfügbare Aufkommen der Biomasseträger Stroh und Waldrestholz lässt sich hinsichtlich ihres Potenzials dann am besten einordnen, wenn man es in Vergleich setzt zu weiteren biogenen Rest- und Abfallstoffen, die ebenfalls für eine energetische Nutzung in Frage kommen (vgl. Abb. 1).

In Deutschland beträgt das jährlich verfügbare Aufkommen (Bezugsjahr: 2002) an biogenen Reststoffen und Abfällen, das energetisch genutzt werden könnte, rd. 70 Mio. Megagramm $(\mathrm{Mg})$ organische Trockensubstanz (oTS); in Baden-Württemberg sind dies rd. 8 Mio. Mg oTS. Betrachtet man die Aufschlüsselung des Aufkommens, so wird deutlich, dass dieses mengenmäßig besonders durch die Land- und Forstwirtschaft bestimmt wird. Auf Bundesebene tragen Stroh, Waldrestholz und Gülle $58 \% \mathrm{zu}$ diesem für eine energetische Nutzung verfügbaren Aufkommen bei; in Baden-Württemberg sind dies $55 \%$.
In Deutschland stehen - gerechnet als Trockenmasse $(\mathrm{TM})$ - rd. 30 Mio. Mg Stroh und Waldrestholz für eine energetische Nutzung zur Verfügung. Gemessen am gesamten Aufkommen biogener Rest- und Abfallstoffe sind dies $43 \%$. Das für eine energetische Nutzung verfügbare jährliche Aufkommen an Stroh und Waldrestholz liegt in BadenWürttemberg bei rd. 3,5 Mio. Mg TM (vgl. Abb. 1 und Abb. 2); dies entspricht wie auf Bundesebene ebenfalls $43 \%$. Bei den relativen Beiträgen von Stroh und Waldrestholz gibt es jedoch deutliche Unterschiede: Im waldreichen Baden-Württemberg trägt Waldrestholz $31 \%$, Stroh aber nur $12 \%$ zum Aufkommen bei; auf Bundesebene sind dies $22 \%$ bzw. $21 \%$.

Das angeführte Aufkommen von 70 Mio. $\mathrm{Mg}$ oTS pro Jahr in Deutschland entspricht einem jährlichen Pro-Kopf-Aufkommen von $0,85 \mathrm{Mg}$ oTS. Gemessen am Heizwert entspricht das rd. 420 Litern Heizöl und damit rd. $9 \%$ des deutschen Primärenergiebedarfs. Zur Einordnung: Im Jahr 2005 deckten Biomasse

Abb. 1: Aufkommen biogener Reststoffe und Abfälle in Deutschland und Baden-Württemberg 2002 verfügbar für eine energetische Nutzung

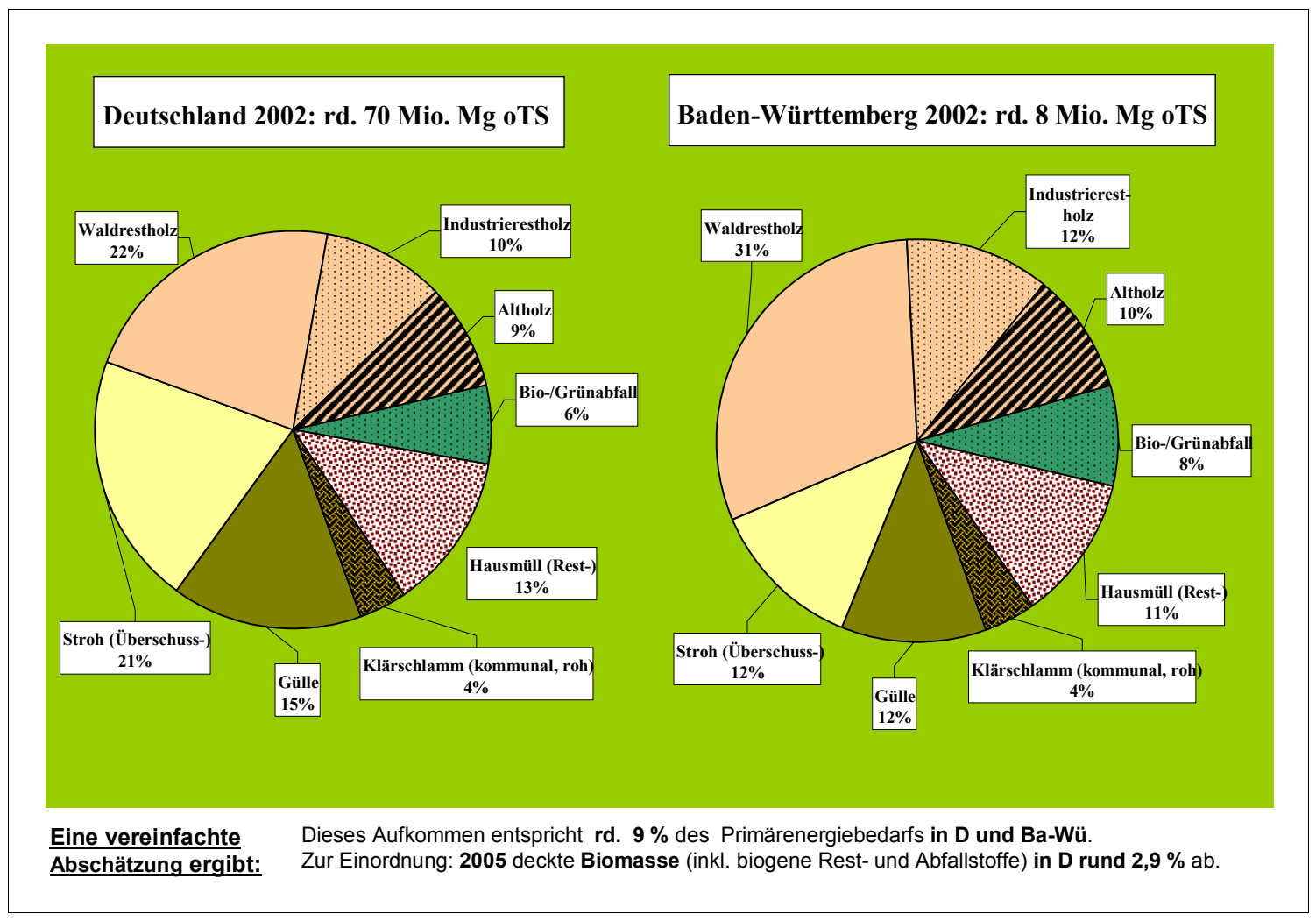

oTS = organische Trockensubstanz 
Abb. 2: Aufkommen und Bereitstellungskosten bei Stroh und Waldrestholz zur Versorgung zweier Anlagenstandorte in Baden-Württemberg

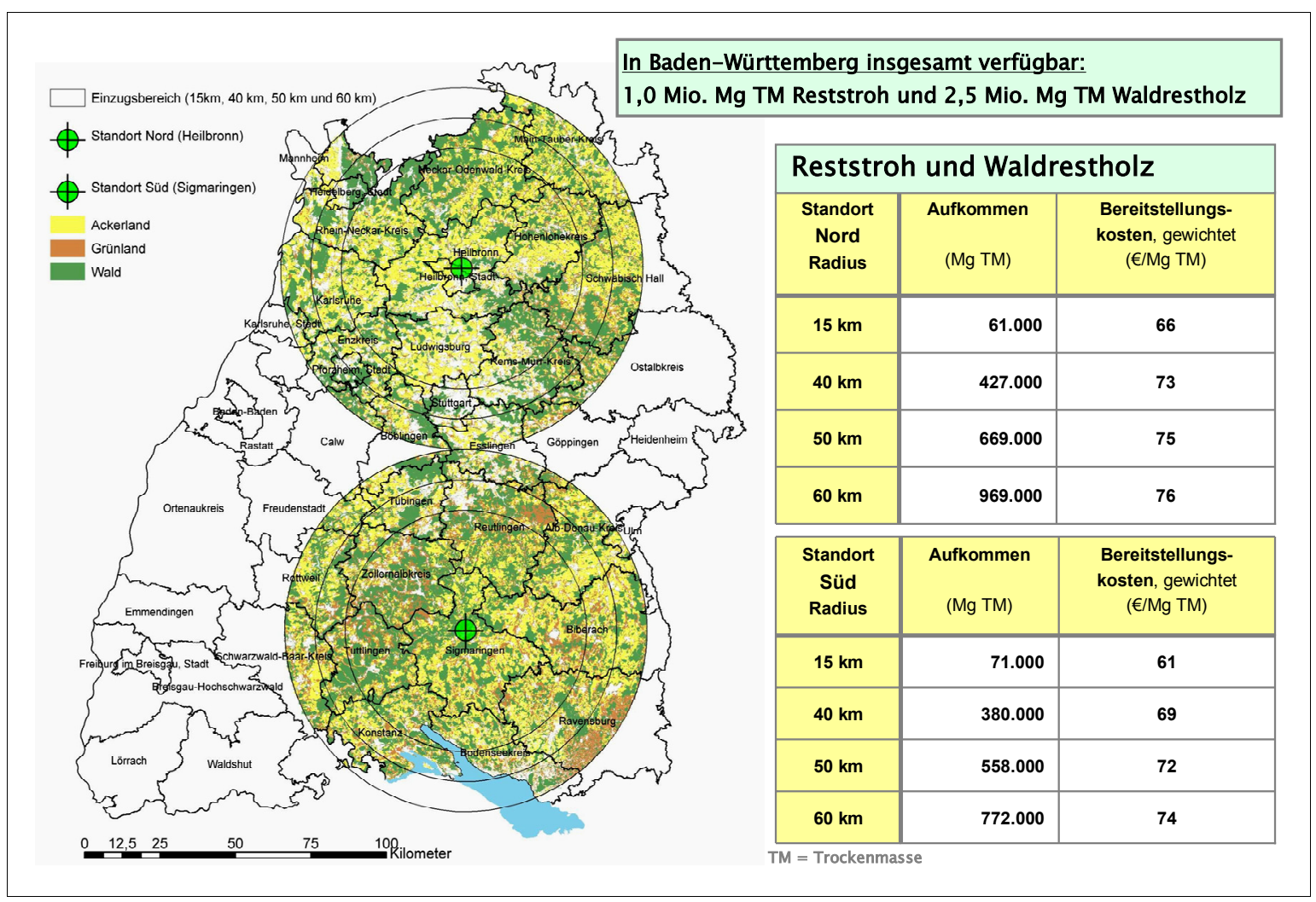

und biogene Rest- und Abfallstoffe in Deutschland rund 2,9\% des Primärenergiebedarfs ab.

Nach diesem allgemeinen Überblick werden nachfolgend für Stroh und Waldrestholz regional differenzierte Ergebnisse zu den Verhältnissen in Baden-Württemberg aus einer aktuellen Untersuchung vorgestellt (vgl. Leible et al. 2005). Hierbei stand die Fragestellung im Mittelpunkt, zu welchen Kosten die Biomasseversorgung mit 1 Mio. Mg TM pro Anlage und Jahr von zwei Anlagenstandorten gewährleistet werden kann. Abbildung 2 gibt hierzu die Ergebnisse für eine Versorgung mit Stroh und Waldrestholz wieder.

Wie die Analysen - unter Einsatz eines geografischen Informationssystems - für die beiden Anlagenstandorte Nord (Heilbronn) und Süd (Sigmaringen) andeuten (s. linker Ausschnitt der Abb. 2), werden mit zunehmender Ausweitung des Erfassungs-Radius (von $15 \mathrm{~km}$ auf 40, 50 und $60 \mathrm{~km}$ ) in sehr unterschiedlichem Maße Ackerland, Grünland- und Waldflächen angeschnitten. Diese
Flächen sind die Basis für die Abschätzung des potenziell verfügbaren Aufkommens an energetisch nutzbarer Biomasse. Die Ergebnisse zeigen, dass ein Erfassungsradius von 60 bis $70 \mathrm{~km}$ ausreichend ist, um eine Versorgung mit jährlich 1 Mio. Mg TM an Stroh und Waldrestholz gewährleisten zu können, bei durchschnittlichen Bereitstellungskosten frei Anlage von $70-80 € / \mathrm{Mg} \mathrm{TM}$.

\section{Energetische Nutzungspfade für Biomasse und biogene Reststoffe}

Betrachtet man die verschiedenen Arten und Herkünfte biogener Energieträger (vgl. Abb. 3 nächste Seite), so können diese prinzipiell nach ihrer Bereitstellung - je nach Art und Konditionierungsform - durch physikalischchemische, bio- oder thermochemische Umwandlungsprozesse in flüssige, gasförmige und feste Energieträger überführt werden.

Hieraus lassen sich dann über die Verbrennung und thermisch-mechanische 


\section{Abb. 3: Bereitstellungswege von Nutzenergie aus biogenen Energieträgern}

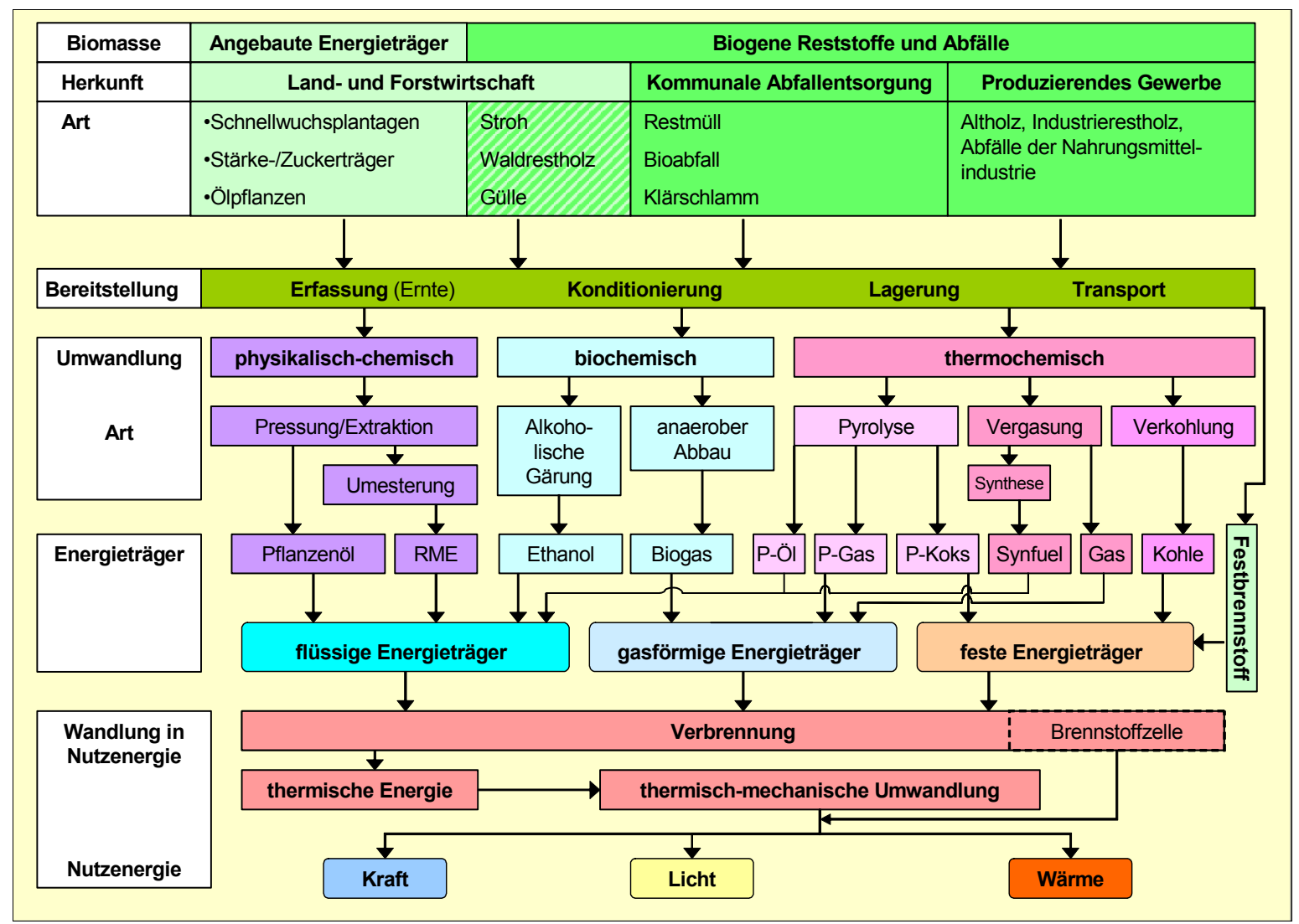

Umwandlung die gewünschten Nutzenergieformen in Form von Kraft, Licht und Wärme bereitstellen. Vor dem Hintergrund der Vielzahl der Prozessschritte ist anzumerken, dass hierbei angestrebt werden sollte, möglichst auf direktem Wege zur gewünschten Nutzenergieform $\mathrm{zu}$ gelangen. Hierdurch werden einerseits die Kosten minimiert und andererseits die Netto-Wirkungsgrade in der Energiebereitstellung optimiert.

\section{Produktion von FT-Kraftstoff aus Stroh und Waldrestholz}

Vor dem Hintergrund des politisch geforderten Ausbaus der energetischen Nutzung von Biomasse verfolgt das Forschungszentrum Karlsruhe mit seinem zweistufigen „Biomass-toLiquid"(BtL)-Konzept das Ziel, aschereiche Biomasse (z. B. Getreidestroh) über die Vergasung für die Bereitstellung synthetischer Kraftstoffe (,Synfuels“) und für eine chemische Nutzung zu erschließen (vgl. Abb. 4, s. auch Malcher et al. 2006). Eine teilweise Verstro- mung der Biomasse bzw. von Zwischenprodukten (z. B. Pyrolysegas) oder eine Wärmegewinnung ist hierdurch nicht ausgeschlossen.

Die Synthese konzentriert sich hierbei zunächst auf FT-Kraftstoffe, ist aber für eine Vielzahl von Produkten offen. Je nach Produkt resultiert die Anforderung, die Synthese bei Drücken von rd. 20 bis 40 bar (für FTKraftstoffe) bzw. bis 80 bar (z. B. für Methanol) durchzuführen. Deshalb wird das Ziel verfolgt, bereits mit der Vergasung das für die Synthese nötige Druckniveau zu erreichen und auch die Gasreinigung und -konditionierung auf dieser Druckstufe zu realisieren. Hierdurch wird der aufwändige Schritt der Gaskompression vor der Synthese vermieden. Darüber hinaus soll ein teerfreies und methanarmes Synthesegas gewonnen werden. Mit einem Flugstromdruckvergaser ist dies bei Vergasungstemperaturen von mehr als $1000{ }^{\circ} \mathrm{C}$ möglich, wie die vom Forschungszentrum auf einer externen Anlage in Freiberg / Sachsen durchgeführten Versuchskam- 
Abb. 4: Zweistufiges Konzept zur Synthesegas- bzw. Kraftstofferzeugung aus Stroh und Waldrestholz

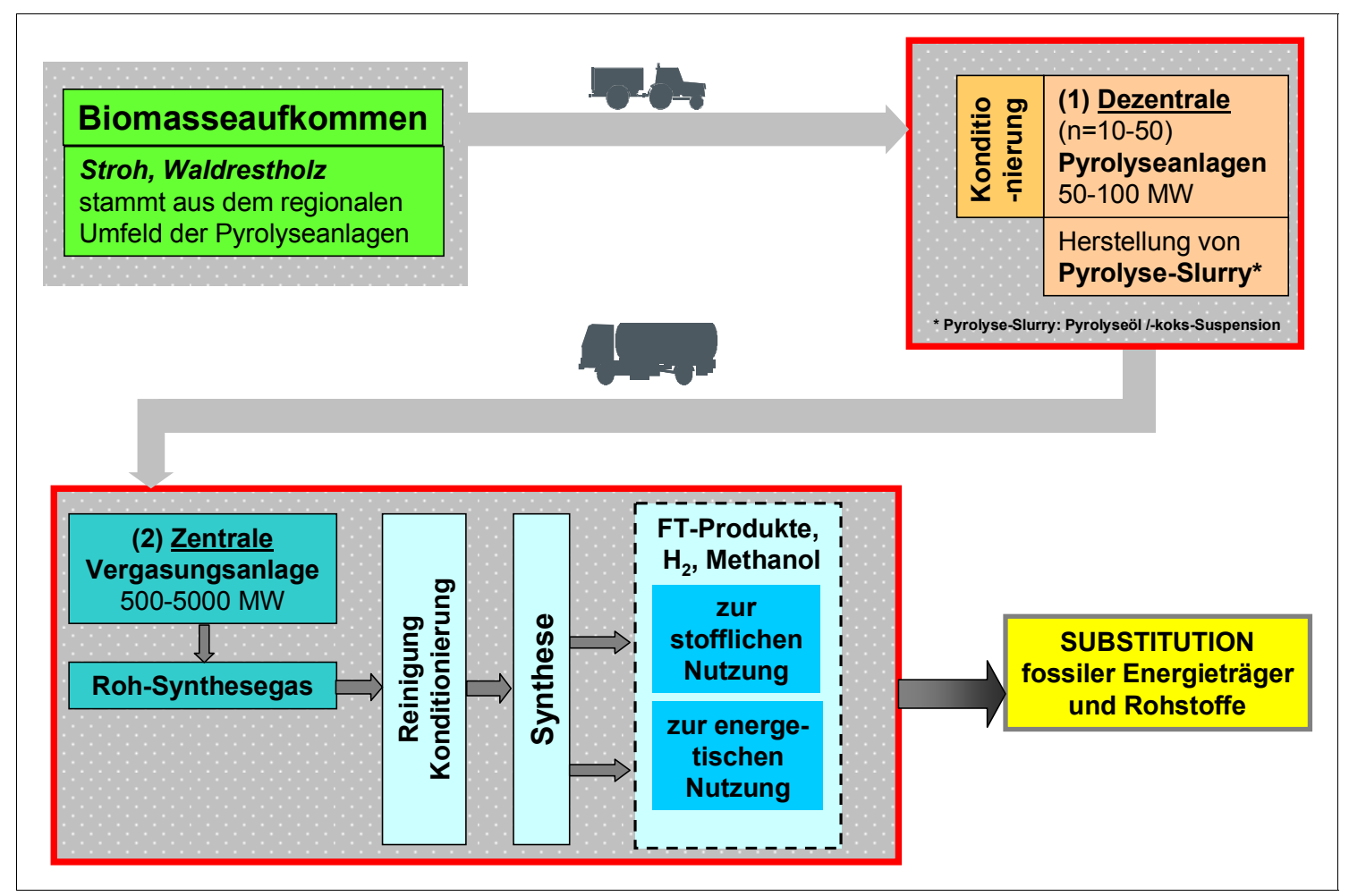

pagnen bestätigten. Für die Einspeisung der Biomasse in den Flugstromdruckvergaser muss diese entsprechend konditioniert werden. Bei den angeführten Drücken ist dies nur über eine pumpbare Suspension (Slurry) aus Pyrolysekondensat und -koks sinnvoll umzusetzen. Folglich ist das vom Forschungszentrum Karlsruhe verfolgte Schnellpyrolyseverfahren zur Herstellung einer solchen Suspension von zentraler Bedeutung (vgl. Lange et al. 2006; Henrich und Dinjus 2005).

Daneben ist mit der räumlichen Entkopplung (siehe Abb. 4) von Schnellpyrolyse und Vergasung (inkl. Gasreinigung/-konditionierung und Synthese) die Option gegeben, eine von der Größe der Vergasungsanlage unabhängige dezentrale Produktion von Slurries zu realisieren. Mit dem anschließenden Transport der Slurries zu einer großen zentralen Vergasungs- und Syntheseanlage lassen sich logistische Vorteile erschließen - verglichen mit Strohballen haben Slurries eine um den Faktor 10 höhere Energiedichte.

Der Vergleich der Kraftstoffproduktion aus Stroh und Waldrestholz bei vorgeschalteter dezentraler Schnellpyrolyse mit der Vari- ante der in einer zentralen Anlage integrierten Schnellpyrolyse ist hierbei von besonderem Interesse, da Transportvorteile bei der dezentralen Variante erhofft werden. Die durchgeführten Analysen zeigen, dass die Produktionskosten für FT-Kraftstoff (Synfuel) aus Stroh - je nach Anlagengröße und dezentralem oder integriertem Konzept - zwischen 105 und $130 € / M W h$ liegen; dies entspricht 1,0 bzw. $1,25 €$ pro Liter (vgl. Abb. 5 nächste Seite). Es fällt auf, dass die Gesamtkosten der Anlagen mit dezentraler Pyrolyse erst ab Anlagengrößen im Bereich von 4.000 MW günstiger werden, verglichen mit der integrierten Pyrolyse. Dies ist so, obwohl bei der integrierten Pyrolyse die Transportkosten für Stroh bei allen Anlagengrößen über der Summe der Transportkosten von Stroh und Slurry bei der dezentralen Pyrolyse liegen. Gewichtiger als dieser Nachteil ist der Vorteil der räumlich integrierten Pyrolyse hinsichtlich der stärkeren Größendegression der Pyrolysekosten und des höheren Wirkungsgrads. 


\section{Abb. 5: Gestehungskosten von FT-Kraftstoff aus Stroh - bei dezentraler oder integrierter Pyrolyse}

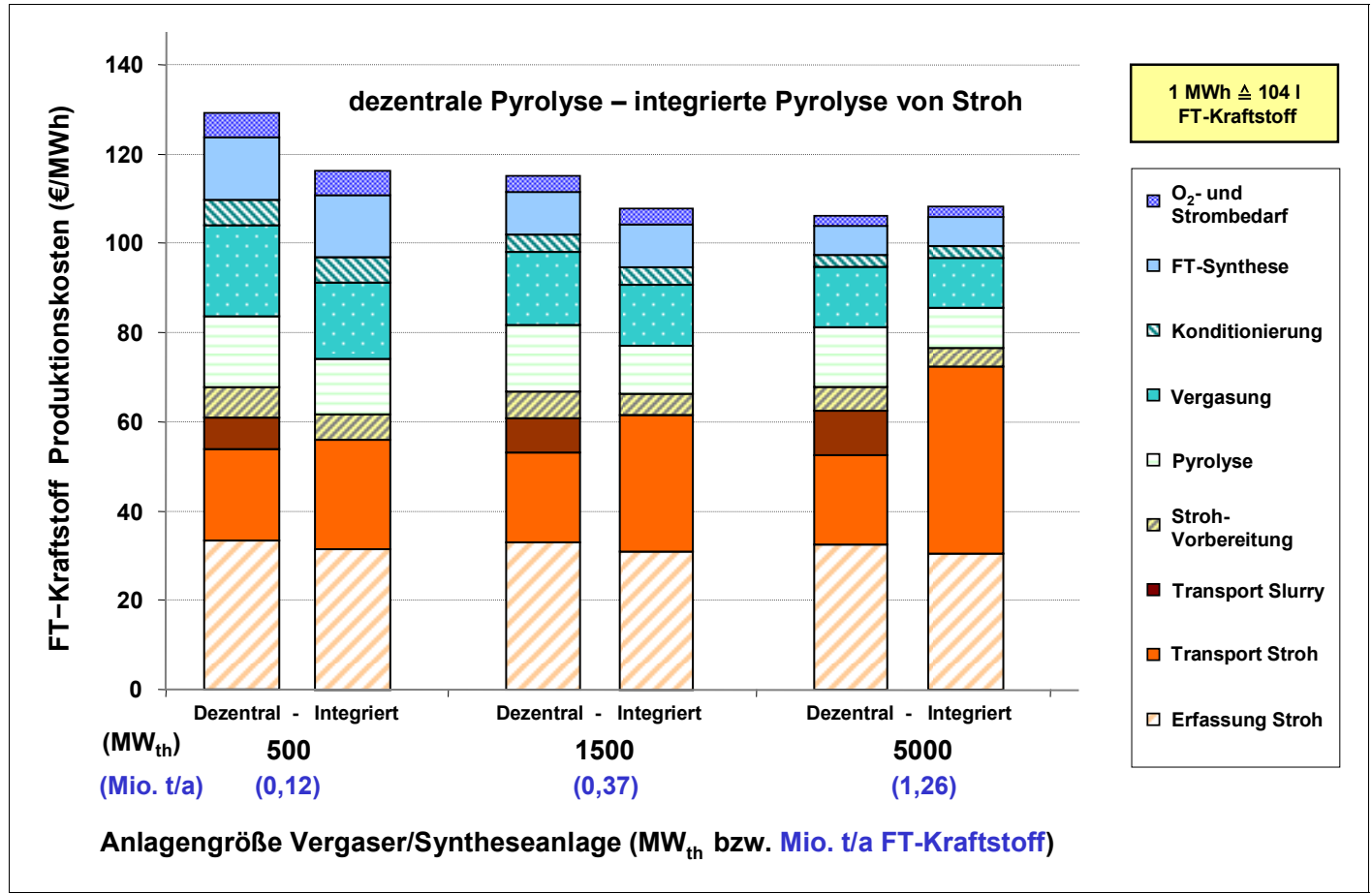

Abbildung 5 verdeutlicht ferner, dass zwei wesentliche Kostenbestandteile unabhängig von der Vergaserleistung sind: die spezifischen Kosten für die Erfassung von Stroh und die für den Lkw-Transport von Stroh bis zur dezentralen Pyrolyseanlage. Bei der integrierten Pyrolyse wird davon ausgegangen, dass der Strohtransport ab einer Entfernung von $100 \mathrm{~km}$ mit der Bahn durchgeführt wird.

Bei höheren verfügbaren Aufkommensdichten an Biomasse treten die Vorteile der integrierten Pyrolyse - wegen der dadurch möglichen Verringerung der Transportstrecken - stärker hervor. Den gleichen Effekt erzielt eine Ausweitung der erfassten Biobrennstoffe auf Waldrestholz. In Abbildung 6 (nächste Seite) sind die Bereitstellungskosten von FT-Kraftstoff aus Stroh und Waldrestholz den Gestehungskosten von Diesel - bei Erdölpreisen von 30, 65 und $100 \$$ pro barrel (bbl) - in einer Raffinerie gegenübergestellt, jeweils ohne Mehrwertsteuer bzw. beim FTKraftstoff auch ohne Mineralölsteuer. Im Ge- gensatz zu Abbildung 5 wurde hier von der gemeinsamen Nutzung von Stroh und Waldrestholz ausgegangen; die dabei nutzbare durchschnittliche Aufkommensdichte (für Deutschland) liegt bei rd. $90 \mathrm{Mg}$ TM pro $\mathrm{km}^{2}$. Als Anlagenkonzept liegt die zentrale Vergasung/Synthese mit integrierter Pyrolyse zugrunde; dabei wurde nach zwei Anlagengrößen mit einer Produktion von 0,2 bzw. 1,0 Mio. Jahrestonnen (jato) unterschieden. Zum Vergleich: Bei herkömmlichen Erdöl-Raffinerien muss eher von 10 Mio. jato an Kraftstoffproduktion ausgegangen werden.

Wie die Ergebnisse zeigen, könnte der FT-Kraftstoff, je nach Anlagengröße, zu rd. $1,00 €$ bzw. $0,90 €$ pro Liter frei Anlage bereitgestellt werden, wenn auf die Mineralölsteuer verzichtet wird. Bei einem Rohölpreis von 65 \$ bbl liegen bei Diesel die Bereitstellungskosten frei Raffinerie - aber einschließlich der Mineralölsteuer - ebenfalls bei rd. $0,90 € / 1$. 
Abb. 6: Gestehungskosten von FT-Kraftstoff aus Stroh und Waldrestholz - ein Vergleich mit fossilem Diesel

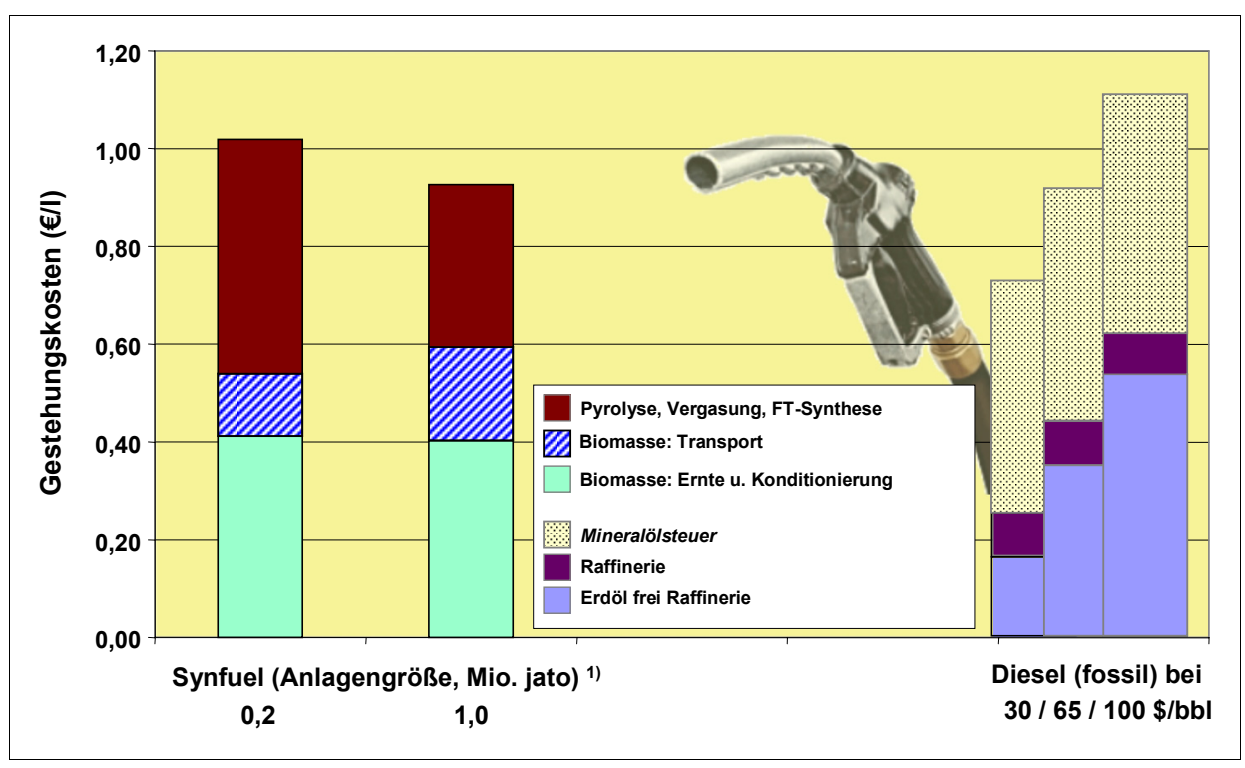

1) Abschätzungen für Synfuel aus Stroh und Waldrestholz, zentrale Anlage; Kostenangaben frei Anlage, vor Steuern

\section{Produktion von Wärme und Strom aus Stroh und Waldrestholz}

Grundsätzlich kann Wärme aus Stroh und Waldrestholz sowohl über die direkte Verbrennung als auch über die Vergasung mit nachgeschalteter Verbrennung bereitgestellt werden. Nachfolgend werden einige Ergebnisse zur Wärmegewinnung über die Verbrennung vorgestellt (vgl. Abb. 7) (vgl. Leible und Kälber 2006). Auf die mit der Stromproduktion gekoppelte Wärmebereitstellung durch Vergasung, z.B. in einem Heizkraftwerk (HKW), soll hier nicht eingegangen werden. Mit Blick auf die Vergasung muss vielmehr festgehalten werden, dass deren klar erkennbaren Vorteile gegenüber der Verbrennung darin gesehen werden, dass in Heizkraftwerken bzw. Kraftwerken höhere elektrische Wirkungsgrade erreichbar sind. Für die reine Wärmeerzeugung ist bei der Vergasung kein prinzipieller Vorteil erkennbar.

Die Wärmegestehungskosten bei den Verbrennungstechnologien, die der alleinigen Wärmeerzeugung dienen, ergeben sich aus den Gesamtkosten der Wärmeerzeugung und sind auf die beim Endverbraucher ankommende Wärmemenge bezogen. Bei der zentralen Wärmeerzeugung im Heizwerk sind hierbei die Investitionen für die Wärmeverteilung und die Verluste bei der Wärmeverteilung berücksichtigt. Bei der gekoppelten Erzeugung von Wärme und Strom ergeben sich die Wärmegestehungskosten aus den Gesamtkosten abzüglich einer Stromgutschrift (Stromerlös, vgl. Abb. 7 nächste Seite). Dieser Gutschrift liegen die Stromgestehungskosten in einem Steinkohlekraftwerk zugrunde.

Die Modellrechnungen zeigen, dass den Kapitalkosten und den Kosten für Stroh und Waldrestholz über den gesamten betrachteten Leistungsbereich der Anlagen eine dominierende Rolle an den Gesamtkosten der Wärmeerzeugung zukommt. Daher werden in Abbildung 7 neben den Wärmegestehungskosten in $€ / M W h$ zusätzlich die prozentualen Anteile der wichtigsten Kostenkomponenten an den Gesamtkosten dargestellt; einbezogen ist dabei der Beitrag der Stromerlöse (im KWK-Betrieb) zur Deckung der Gesamtkosten. 


\section{Abb. 7: Wärmegestehungskosten und Kostenanteile bei verschiedenen Verbrennungstechnologien für} Holz und Stroh

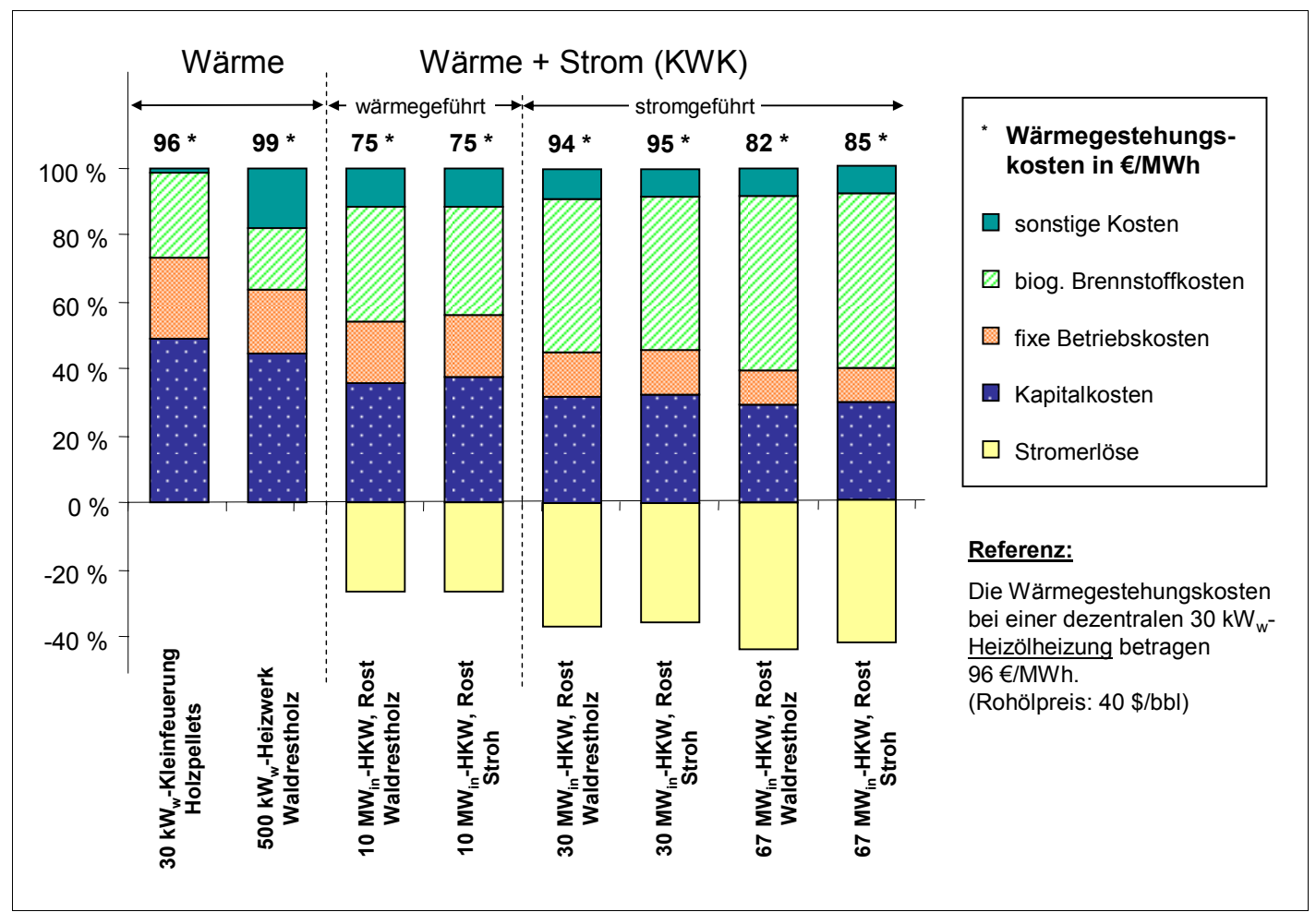

$\mathrm{kW}_{\mathrm{w}}=$ Nennwärmeleistung

$\mathrm{MW}_{\text {in }}=$ Brennstoffleistung Input

Wie anhand der Ergebnisse zu sehen ist, reduzieren sich die Wärmegestehungskosten mit steigender Anlagengröße - von $96 € /$ MWh bei der $30 \mathrm{~kW}-$ Kleinfeuerung (Holzpellets aus Industrierestholz) auf $75 € /$ MWh beim wärmegeführten $10 \mathrm{MW}_{\text {in }}{ }^{1}$ Heizkraftwerk. Vergleicht man dies mit den Wärmegestehungskosten einer $30 \mathrm{~kW}$-Heizölfeuerung von $96 € / \mathrm{MWh}$ - hierbei liegt ein Rohölpreis von $40 \$$ pro barrel zugrunde -, so ist die Wärmeerzeugung auf Basis von Waldrestholz oder Stroh im wärmegeführten Heizkraftwerk eindeutig als wettbewerbsfähig einzustufen. Unter diesen Rahmenbedingungen hat die dezentrale Wärmeerzeugung in einer Kleinfeuerung $(30 \mathrm{~kW})$, die mit Holzpellets aus Industrierestholz befeuert wird, im Vergleich zu einer Heizöl-Referenzanlage gerade die Wettbewerbsfähigkeit erreicht - auch ohne Investitionszulagen (vgl. Abb. 7). Dauerhaft höhere Rohölpreise im Bereich von 50 bis $60 \$$ pro barrel werden insbesondere in ländlichen Gebieten die Zunahme der Wärmeerzeugung aus Biomasse deutlich beflügeln.
Im Bereich der Kraft-Wärme-Kopplung zeigt sich beim Sprung vom wärme- zum stromgeführten Betrieb - trotz zunehmender Anlagengröße - ein Anstieg der Wärmegestehungskosten. Dies begründet sich aus der unterstellten Abnahme der Wärmevolllaststunden beim Übergang vom wärme- zum stromgeführten Betrieb. Im wärmegeführten Betrieb werden noch 6.000 Volllaststunden pro Jahr angenommen, beim stromgeführten Betrieb verringert sich die Anzahl der Volllaststunden auf 4.000 pro Jahr. Beim Leistungssprung auf ca. $67 \mathrm{MW}_{\text {in }}$ ergibt sich dann wieder das aufgrund der Kostendegression erwartete Bild abnehmender Wärmegestehungskosten. Der Einsatz der unterschiedlichen Brennstoffe Waldrestholz und Stroh wirkt sich im Bereich der Kraft-WärmeKopplung eher unwesentlich aus.

Aus Abbildung 7 wird darüber hinaus die grundsätzliche Beziehung deutlich, dass mit zunehmender Anlagengröße der Anteil der Brennstoffkosten an den Gesamtkosten ansteigt und bei großen Anlagen mit bis zu $60 \%$ die Gesamtkosten dominiert. Gegenläufig dazu 
nehmen der Einfluss der Kapitalkosten - aufgrund der Degression bei den spezifischen Investitionen - und die Bedeutung der Personalkosten deutlich ab.

Mit Blick auf die Bereitstellung von Strom aus Stroh und Waldrestholz wurde eine Vielzahl höchst heterogener Technologien untersucht (vgl. Leible und Kälber, 2006). In Abbildung 8 werden die Stromgestehungskosten für ausgewählte Technologien in Abhängigkeit von der elektrischen Anlagenleistung dargestellt.

Als Vergleich dienen die Stromgestehungskosten in einem mit Importkohle betriebenen Steinkohlekraftwerk, die bei rd. $52 € /$ $\mathrm{MWh}_{\mathrm{el}}$ liegen, und der Bereich für die Stromvergütung nach dem Erneuerbare-EnergienGesetz (EEG 2000 / 2004) für die Einspeisung von Strom aus Biomasse. Durch die Novellierung des EEG - gültig seit August 2004 - hat sich die Vergütung für regenerativen Strom deutlich verbessert. Ergänzend ist an dieser Stelle anzuführen, dass die Stromgestehungskosten mit inländischer Steinkohle - nicht subventioniert - bei rd. $80 € / \mathrm{MWh}_{\mathrm{el}}$ liegen. Andererseits kann derzeit in einem nahezu vollständig abgeschriebenen Steinkohlekraftwerk mit Importkohle Strom zu rd. $33 € / \mathrm{MWh}_{\mathrm{el}}$ bereitgestellt werden.

Die Verbrennung und Vergasung von Stroh und Waldrestholz zur Stromgewinnung sind gegenwärtig i.d. R. nicht wettbewerbsfähig. Dies liegt insbesondere an den hohen Kosten der Biomassebereitstellung. Die Co-Verbrennung (Zufeuerung) von Waldrestholz und Stroh im Steinkohlekraftwerk stellt eine vergleichsweise kostengünstige Möglichkeit dar, den fossilen Brennstoff Steinkohle zumindest teilweise zu substituieren. Die Stromgestehungskosten liegen hier zwischen 90 und $95 € / \mathrm{MWh}_{\mathrm{el}}$ (vgl. Abb. 8). Obwohl die Datenbasis und die darauf aufbau-

\section{Abb. 8: Stromgestehungskosten bei der energetischen Nutzung von Stroh und Waldrestholz}

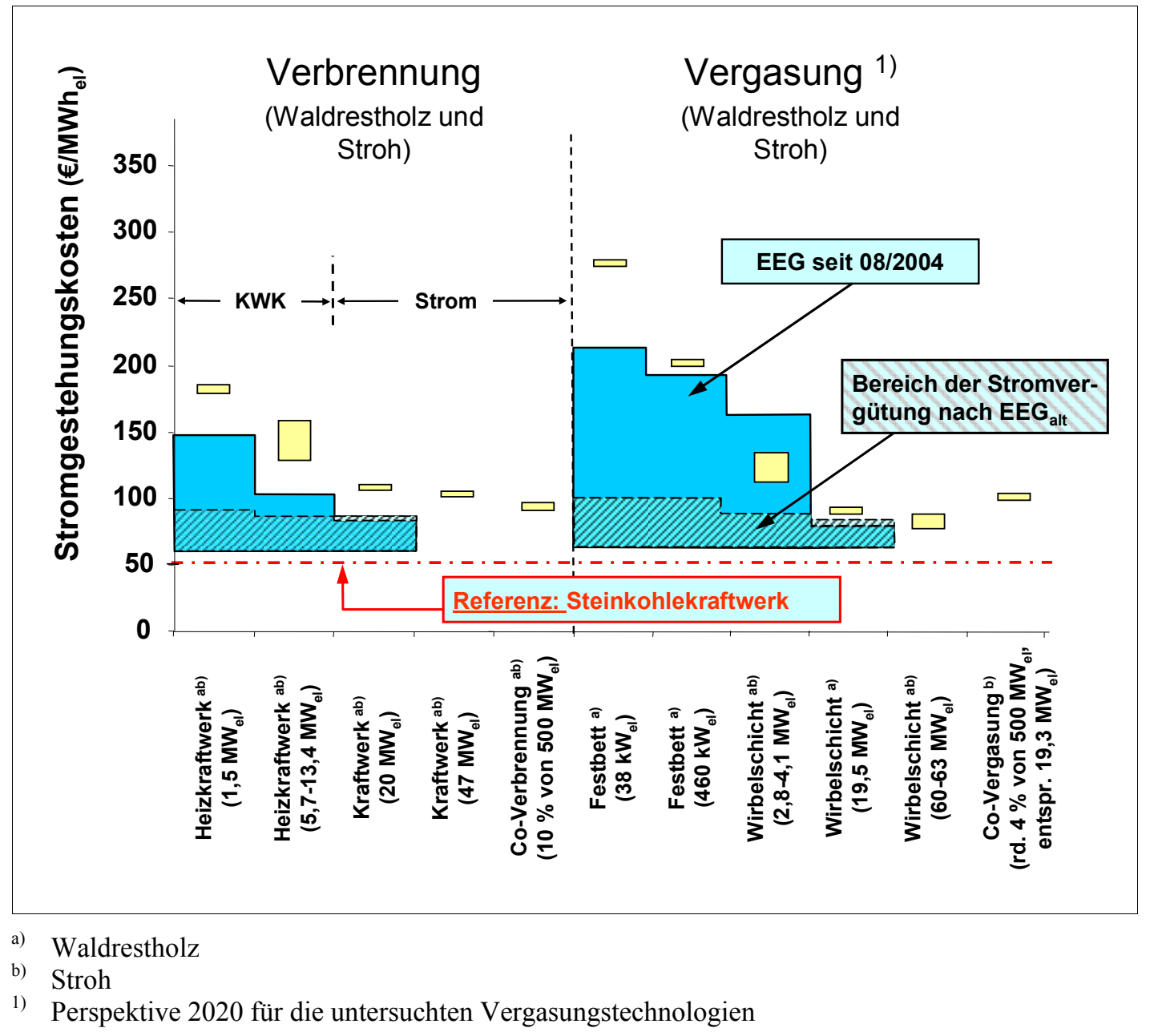


ende Bewertung der Vergasungstechnologien mit einer hohen Unsicherheit behaftet sind, lassen sich mit Vorbehalten einige Schlussfolgerungen ziehen. So sind beispielsweise bei den Stromgestehungskosten Vorteile für Vergasungsanlagen ab etwa $3 \mathrm{MW}_{\mathrm{el}}$ erkennbar, gegenüber den direkten Verbrennungstechnologien.

\section{Vergleich der Kraftstoff- mit der Wärme- und Stromproduktion}

Als konkurrierende Verfahren für die Nutzung von Stroh und Waldrestholz wurden einerseits die Wärme- und Stromgewinnung durch direkte Verbrennung in Biomasse-Heizwerken bzw. Biomasse(heiz)kraftwerken und die CoVerbrennung in Steinkohlekraftwerken berücksichtigt; dies schloss die thermochemische Vergasung zur Stromerzeugung mit ein. Andererseits wurden die auf fossilen Energieträgern (Rohöl, Import-Steinkohle) basierenden Alternativen der Wärme-, Strom- und Kraftstofferzeugung dargestellt.

In Abbildung 9 werden nun die Wärme-, Strom- und Kraftstoffgestehungskosten für die betrachteten Verfahren einander gegenübergestellt. Als fossile Referenzen dienen, wie be- reits erwähnt, die Wärmegestehungskosten in einer mit Heizöl betriebenen Kleinfeuerung diese liegen derzeit bei rund $96 € / \mathrm{MWh}_{\mathrm{w}}-$, die Stromgestehungskosten in einem Steinkohlekraftwerk $\left(500 \mathrm{MW}_{\mathrm{el}}\right)$ - diese liegen bei rd. $52 € / \mathrm{MWh}_{\mathrm{el}}-$ und die Bereitstellungskosten von Dieselkraftstoff, die in einem Bereich von 30 bis $45 € /$ MWh liegen, je nach unterstelltem Erdölpreis.

Beim Vergleich der FT-Kraftstoffgewinnung mit der Wärmeerzeugung aus Stroh und Waldrestholz wird deutlich, dass die Wärmeerzeugung näher an der Wettbewerbsfähigkeit ist bzw. diese bereits erreicht hat. So zeigen die Ergebnisse, dass bereits heute die Wärmebereitstellung in der Regel nahezu ohne Subventionen auskommt. Dem gegenüber hat die FT-Kraftstoff-Produktion einen deutlichen Subventionsbedarf. Eine wirtschaftlich konkurrenzfähige Produktion von FT-Kraftstoffen ohne Mineralölsteuerverzicht wäre erst bei Rohölpreisen von deutlich über 100 \$ pro barrel möglich.

Mit der Substitution fossiler Energieträger durch erneuerbare kann die Emission treibhausrelevanter Gase und somit der Treibhauseffekt deutlich reduziert werden. Bei den durchgeführ-

Abb. 9: Gestehungskosten von Wärme, Strom und Kraftstoff aus Stroh und Waldrestholz

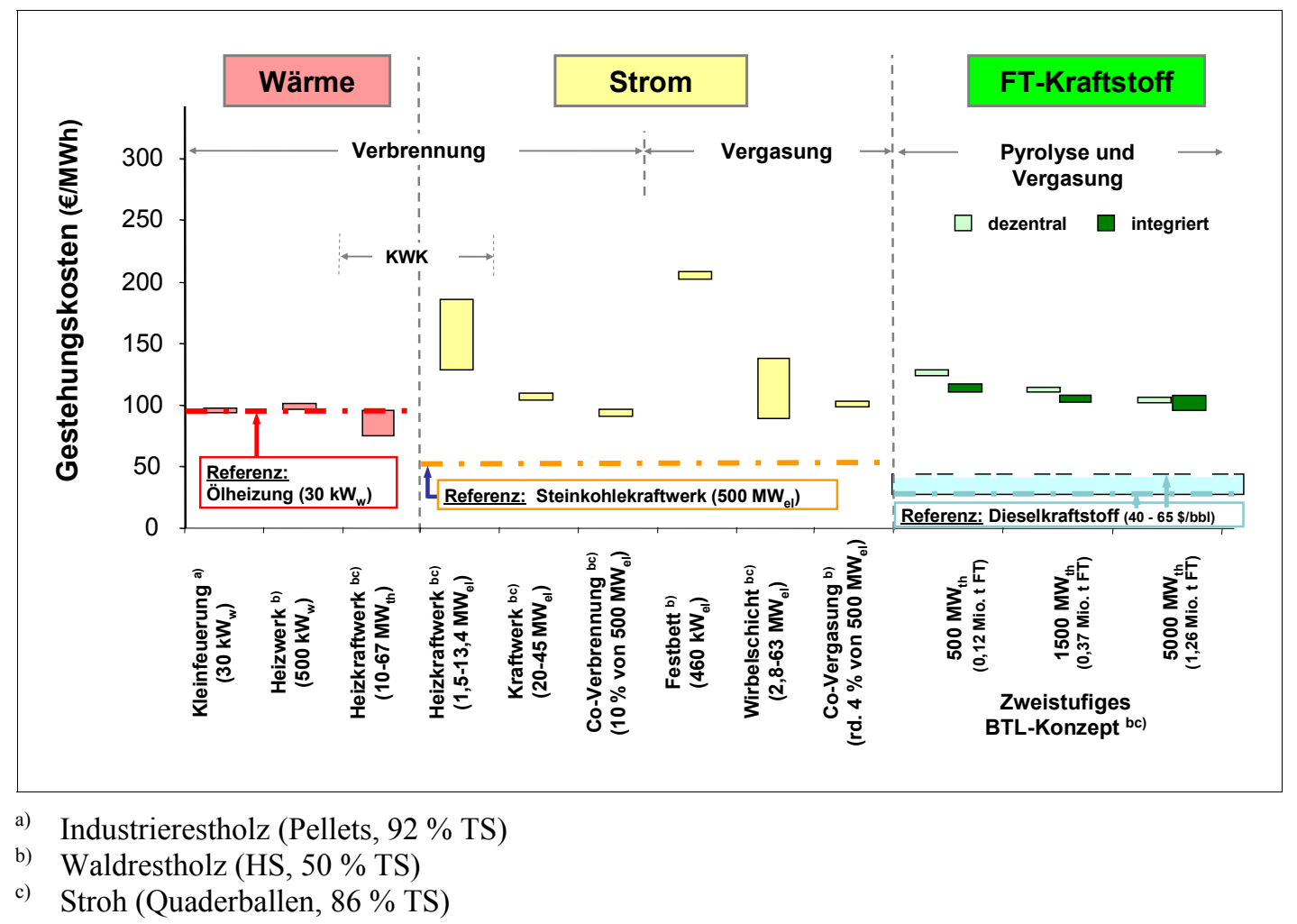




\section{Abb. 10: $\mathrm{CO}_{2}$-Minderungskosten bei der Gewinnung von Wärme, Strom und Kraftstoff aus Stroh und} Waldrestholz

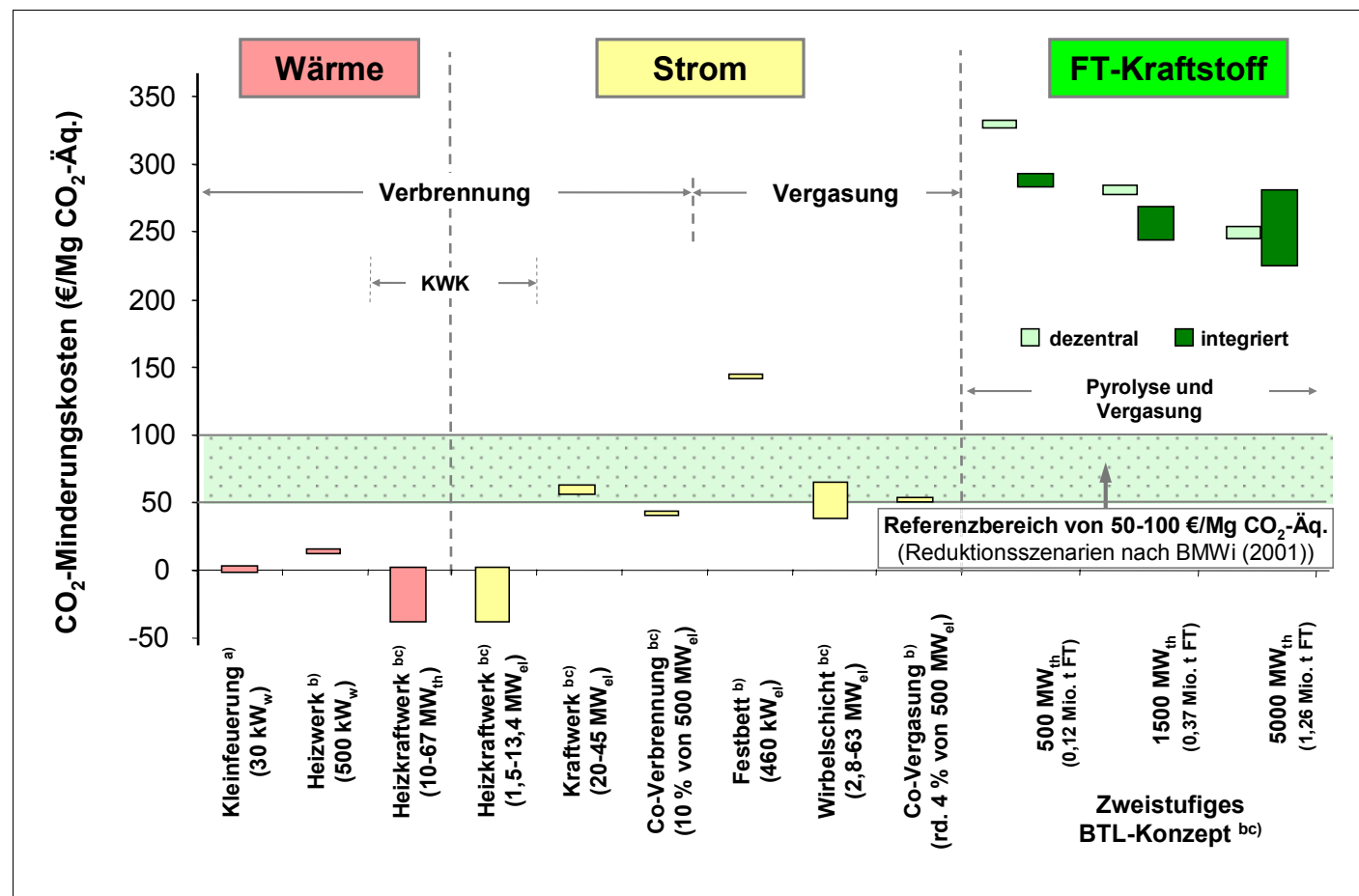

a) Industrierestholz (Pellets, $92 \% \mathrm{TS}$ )

b) Waldrestholz (HS, $50 \% \mathrm{TS}$ )

c) Stroh (Quaderballen, $86 \%$ TS)

ten Analysen wurden neben $\mathrm{CO}_{2}$ auch die Treibhausgase $\mathrm{CH}_{4}$ (Methan) und $\mathrm{N}_{2} \mathrm{O}$ (Lachgas) einbezogen und in der Summe als $\mathrm{CO}_{2}$-Äquivalente $\left(\mathrm{CO}_{2}\right.$ - ̈̈q.) dargestellt (vgl. Abb. 10). Die $\mathrm{CO}_{2}$-Minderungskosten ergeben sich aus den Mehrkosten auf der einen Seite und der erzielten $\mathrm{CO}_{2}$-Minderung gegenüber der fossilen Referenz auf der anderen Seite. Mit ihrer Hilfe kann dargestellt werden, wie teuer die jeweilige Technologie bei der Verfolgung einer $\mathrm{CO}_{2-}$ Minderungsstrategie ist. Zur vergleichenden Bewertung wurden $\mathrm{CO}_{2}$-Minderungskosten aus Studien mit $\mathrm{CO}_{2}$-Minderungsszenarien bei der Verfolgung der Minderungsziele der Bundesregierung herangezogen (vgl. BMWI 2001). Aussagen aus diesen Studien ergeben, dass bei einem $\mathrm{CO}_{2}$-Minderungsziel von $25 \%$ oder gar von $40 \% \mathrm{CO}_{2}$-Minderungskosten zwischen 50 und $100 €$ pro $\mathrm{Mg} \mathrm{CO}_{2}$ - $\mathrm{Äq}$. angesichts teurerer Alternativen durchaus zu akzeptieren sind.

Bei der Produktion von FT-Kraftstoffen aus Stroh und Waldrestholz liegen die $\mathrm{CO}_{2}$ Minderungskosten deutlich über $200 € / \mathrm{Mg}$ $\mathrm{CO}_{2}$-Äquivalent. Bei der Verstromung - mit
Ausnahme der Festbettvergasung - resultieren Kosten von unter $100 € / \mathrm{Mg} \mathrm{CO}_{2}$-Äquivalent. Am günstigsten lässt sich die $\mathrm{CO}_{2}$-Minderung über die Wärmebereitstellung realisieren. Hier fallen nahezu keine bzw. sogar negative $\mathrm{CO}_{2}$ Minderungskosten an.

Die Abschätzungen $\mathrm{zu}$ den $\mathrm{CO}_{2}$-Minderungskosten verdeutlichen, dass die $\mathrm{CO}_{2}$-Minderungsstrategie nur ein sehr schwaches Argument für die Forcierung der Aktivitäten zur Bereitstellung von FT-Kraftstoffen aus Biomasse darstellen kann.

Da das BtL-Konzept des Forschungszentrums Karlsruhe über die Pyrolyse und Vergasung Wege eröffnet, die Biomasse - als Kohlenstoffträger - einer weitergehenden chemischen Nutzung zuzuführen, sollte dieser Entwicklungsweg weiter beschritten werden. Dies schließt eine gekoppelte chemisch/energetische Nutzung im Sinne eines „Biorefinery“-Konzepts mit ein. Darüber hinaus lassen sich unter Nutzung des technischen Fortschritts die bestehenden ökonomischen Nachteile bei der Kraftstofferzeugung sicherlich reduzieren, so dass 
insbesondere unter Vorsorge-Gesichtspunkten die Forschung und Demonstration in diesem Bereich intensiviert werden sollten.

\section{Anmerkung}

1) Durch die Indizierung der Anlagenleistung in Form von $\mathrm{MW}_{\text {in }}$ (=Inputleistung Brennstoff) soll eine klare Unterscheidung gegenüber Anlagen mit ausschließlicher Wärmeerzeugung vorgenommen werden; bei diesen wird von der Nennwärmeleistung (z. B. $\mathrm{kW}_{\mathrm{w}}$ ) gesprochen.

\section{Literatur}

BMWi - Bundesministerium für Wirtschaft und Technologie (Hg.), 2001: Energiepolitische und gesamtwirtschaftliche Bewertung eines $40 \%$ igen Reduktionsszenarios. Endbericht von Prognos, EWI und BEI, Juli 2001. Gutachten erstellt im Auftrag des BMWi, Dokumentation Nr. 492, Berlin, 79 S. + Anhang

Henrich, E.; Dinjus, E., 2005: Die PyrolyseslurryVergasung des Forschungszentrums Karlsruhe. In: Fachagentur Nachwachsende Rohstoffe (Hg.), Schriftenreihe Nachwachsende Rohstoffe, Band 25 „Synthetische Biokraftstoffe - Techniken, Potenziale und Perspektiven. Münster: Landwirtschaftsverlag, S. 236-268

Lange, S., Reimert, R.; Leible, L., 2006: Systemanalyse zur Schnellpyrolyse als Prozessschritt bei der Herstellung von Synthesekraftstoffen aus Stroh und Waldrestholz. In: DGMK (Hg.): Proceedings zur Fachbereichstagung „Energetische Nutzung von Biomassen". Hamburg: DGMK (in Druck)

Leible, L.; Kälber, S., 2006: Energetische Nutzung fester biogener Reststoffe. In: Bundesamt für Bauwesen und Raumordnung (Hg.): „Bioenergie: $\mathrm{Zu}-$ kunft für ländliche Räume“. Informationen zur Raumentwicklung, Heft 1+2 (2006), S. 43-54

Leible, L.; Kälber, S.; Kappler, G., 2005: Entwicklungen von Szenarien über die Bereitstellung von land- und forstwirtschaftlicher Biomasse in zwei baden-württembergischen Regionen zur Herstellung von synthetischen Kraftstoffen - Mengenszenarien zur Biomassebereitstellung, Abschlussbericht. Forschungszentrum Karlsruhe http://www.itas.fzk.de/ deu/lit/2005/leua05a.pdf

Malcher, L.; Henrich, E.; Leible, L.; Wiemer, H.-J., 2006: Gaserzeugung aus Biomasse, Kurzfassung des Abschlussberichts. Karlsruhe http://www.itas.fzk.de/ deu/lit/2006/leua06a_kurzfassung.pdf

\section{Kontakt}

Dr. Ludwig Leible

Institut für Technikfolgenabschätzung und Systemanalyse (ITAS)

Forschungszentrum Karlsruhe in der HelmholtzGemeinschaft

Hermann-von-Helmholtz-Platz 1

76344 Eggenstein-Leopoldshafen

Tel.: +49 (0) 7247 / 82 - 4869

Fax: +49 (0) 7247 / 82 - 4806

E-Mail: leible@itas.fzk.de 